\title{
Droplet digital PCR detects high rate of TP53 R249S mutants in cell-free DNA of middle African patients with hepatocellular carcinoma
}

\author{
Agnès Marchio ${ }^{1}$ Marie Amougou Atsama ${ }^{2}$. Aubin Béré ${ }^{3}$. Narcisse-Patrice Komas ${ }^{4}$. Dominique Noah Noah ${ }^{5}$. \\ Paul Jean Adrien Atangana ${ }^{6}$. Serge-Magloire Camengo-Police ${ }^{7} \cdot$ Richard Njouom $^{2} \cdot$ Claudine Bekondi $^{3}$. \\ Pascal Pineau' ${ }^{1}$ (B)
}

Received: 2 February 2018 / Accepted: 4 May 2018 / Published online: 10 May 2018

(c) The Author(s) 2018

\begin{abstract}
Hepatocellular carcinoma (HCC) is still a major killing malignancy in sub-Saharan Africa. Lifelong intoxication with aflatoxin $\mathrm{B} 1$ is considered as one of the primary causes of this situation. The role of aflatoxin in HCC from a given population is commonly estimated through the prevalence of R249S mutation of TP53, a hallmark for previous exposure to the mycotoxin. However, the role of AFB1 is barely known in large part of Africa. We conducted a survey on circulating cell-free DNA from 149 patients with HCC and 213 control subjects with and without liver diseases from Cameroon and Central African Republic using droplet digital PCR technique. We observed a mutation prevalence of $24.8 \%(n=37 / 149)$ in patients with tumor and $5.6 \%(n=12 / 213)$ in controls $(P=2.2 \mathrm{E}-07)$. Patients with mutations usually displayed significantly increased circulating alpha-fetoprotein (AFP) values, high hepatitis B virus (HBV) DNA loads as well as worsened values of blood cells count. Interestingly, the fraction of droplets positive for R249S was significantly larger in patients with liver cancer $(15.3 \pm 3.7 \%)$ than in controls $(0.5 \pm 0.3 \%, P=7.1 \mathrm{E}-04)$. Our survey indicates that AFB1 is instrumental for HCC development in Middle Africa and that droplet digital PCR might be used in the region both to diagnose HCC and to conduct public health surveys on populations at risk of chronic aflatoxin intoxication.
\end{abstract}

Keywords Hepatocellular carcinoma $\cdot$ Cell-free DNA · Droplet digital PCR · TP53 mutants

Pascal Pineau

pascal.pineau@pasteur.fr

Agnès Marchio

agnes.marchio@pasteur.fr

Marie Amougou Atsama

marieamougou164@yahoo.com

Aubin Béré

berostau@yahoo.fr

Narcisse-Patrice Komas

npkomas@yahoo.fr

Dominique Noah Noah

noahnoahd@yahoo.fr

Paul Jean Adrien Atangana

atangana@pasteur-yaounde.org

Serge-Magloire Camengo-Police camengop@netcourrier.com

Richard Njouom njouom@pasteur-yaounde.org
Claudine Bekondi

claudinebekondi@yahoo.fr

1 Unité “Organisation Nucléaire et Oncogenèse," INSERM U993, Institut Pasteur, 28, rue du Docteur Roux, 752724 Paris Cedex 15, France

2 Service de Virologie, Centre Pasteur du Cameroun, Yaoundé, Cameroun

3 Unité de Rétrovirologie et Virus Oncogènes, Institut Pasteur de Bangui, Bangui, Central African Republic

4 Laboratoire des Hépatites Virales, Institut Pasteur de Bangui, Bangui, Central African Republic

5 Service de Gastro-Entérologie, Hôpital Central, Yaoundé, Cameroun

6 Laboratoire d'Anatomo-Pathologie, Centre Pasteur du Cameroun, Yaoundé, Cameroun

7 Service de Gastro-Entérologie, Hôpital de l'Amitié, Bangui, Central African Republic 


\section{Abbreviations}

$\begin{array}{ll}\text { AFB1 } & \text { Aflatoxin B1 } \\ \text { CAR } & \text { Central African Republic } \\ \text { cfDNA } & \text { Cell-free DNA } \\ \text { ddPCR } & \text { Droplet digital PCR } \\ \text { HCC } & \text { Hepatocellular carcinoma }\end{array}$

\section{Background}

Primary liver cancer (PLC) is currently the second cause of death from malignancy in humans [1]. This prominence is primarily due to the large variety of etiological agents capable of triggering liver tumorigenesis, to the high prevalence of some of them, to the clinically insidious character of liver tumors until an advanced stage, to the absence of universal biomarkers enabling an early detection of the tumor, and finally to the lack of easily implementable curative therapeutics particularly in poor countries where PLC incidence is high.

Hepatocellular carcinoma (HCC), the major histological form of primary liver cancer, is known for a long time for its remarkable geographical variations of incidence [2]. These variations are primarily attributable to the congruent distribution of some major risk factors of HCC. Indeed, in sub-Saharan Africa (SSA), two of them, i.e., chronic infection with hepatitis B virus (HBV) and intoxication with the carcinogenic mycotoxin, aflatoxin B1 (AFB1), are known to be highly prevalent $[3,4]$. As a consequence, HCC is frequently in SSA the first incident tumor common to both sexes just behind breast and cervix carcinomas in women or prostate carcinoma in men [1]. In addition, HCC in SSA has the afflicting peculiarity to develop earlier in life span than in developed countries with patients frequently presenting in their forties, whereas in Europe, it usually takes two additional decades to declare [5].

AFB1, often present in staple foods of populations living in SSA, is known to induce a specific $\mathrm{G}>\mathrm{T}$ mutation (in exon 7, nucleotide 747, hg38 position on Chr17: 7674216), changing an arginine for a serine at codon 249 of the p53 protein (TP53 R249S) [6]. This specific mutation is frequently found in tumor DNA from patients living South-Eastern Asia or sub-Saharan Africa but is rare in tumors of patients coming from other regions of the world [7-9]. In some occasions, TP53 R249S has even been found in more than half of the tumors investigated. The early detection of this frequent alteration might, thus, represent a promising tool for the screening of patients living in the intertropical areas where AFB1 is abundant [10].

The presence of cell-free DNA (cfDNA) in serum is known since 1940 decade [11], and since then, a large number of studies have reported that patients undergoing various types of tumor display higher concentrations of cfDNA in serum/plasma [12, 13]. CfDNA has, therefore, received special attention because of its potential application as a noninvasive, rapid, and sensitive tool for molecular diagnosis and monitoring of cancer. Interestingly, genetic or epigenetic alterations specific to tumor DNA have been retrieved in the plasma of patients diagnosed with different types of cancers [14]. CfDNA analysis was reported to be as specific and sensitive that tissue biopsy for metastatic colorectal cancer [15]. It is currently considered as a target of choice in "liquid biopsies" by some authors and might, therefore, represent a future common source of material for molecular analyses conducted in the frame of routine monitoring of cancer and pre-cancer patients.

Although HCC is a worrying public health problem in Central Africa, its incidence is considered as significantly lower than in West Africa [16]. The reasons explaining such difference are unknown but may be related to a lesser exposure of the Central African populations to AFB 1 or to difference in endemicity of persistent HBV infection. Contrasting with the significant knowledge accumulated about TP53 R249S in West Africa, nothing is currently known about this tumor marker in the nine countries of Central Africa (Chad, Central African Republic, Cameroun, Gabon, Equatorial Guinea, Congo, Democratic Republic of Congo, Sao Tome e Principe, and Angola), a vast region (6.6 million square $\mathrm{km}$ ) peopled with 138 million habitants. We conducted a prospective study on plasma from patients with HCC and controls with or without liver disease to assess the prevalence and potential usefulness of the TP53 R249S biomarker in Cameroun and Central African Republic (CAR), two countries located in Central Africa.

\section{Methods}

\section{Aim, design, and setting}

The aim of the study was to measure the importance of aflatoxin B1-associated mutation of the p53-encoding gene (TP53) in hepatocellular carcinoma from patients of Middle Africa, a hitherto unexplored region. All participants were recruited in a single institution of Cameroun and Central African Republic. The study was designed as a case-control study comparing patients with a hepatocellular carcinoma (HCC) considered as cases, with patients with chronic liver diseases (CLD) but without liver cancer. In addition, a third group of subjects, patients without any liver disease, were taken to provide a baseline for the prevalence of TP53 mutations in Cameroonians and Central Africans. 


\section{Patients}

The current work is a case-control study. Cases were represented by patients affected with HCC consecutively enrolled in the Gastroenterology and Radiology Departments of the Central Hospital, Yaoundé, Cameroon, and in the Department of Hepato-Gastroenterology at the Hôpital de l'Amitié in Bangui, CAR, between February 2013 and January 2014. Cases were individually 1:1 paired-matched by sex and age ( \pm 5 years) with control subjects consecutively selected and represented by patients with or without serological signs of chronic liver infection attending at the same period in the same medical departments.

The diagnosis of $195 \mathrm{HCC}$ cases was based on suggestive clinical symptoms, presence of a liver mass at ultrasound, viral context, and, when possible, histology of tissues samples together with measurement of serum alpha-fetoprotein (AFP) levels. Inclusion criteria for control subjects with a known chronic liver disease (CLD, $n=263$ ) were the absence of liver mass at ultrasound and a normal level of serum AFP $(<10 \mathrm{ng} / \mathrm{mL})$. Patients with healthy liver (HL, $n=49$ ) were recruited in the same medical departments where they were followed for a non-liver affection. All the cases and controls were interviewed using a standardized questionnaire to collect information about demographic characteristics, personal medical history, familial history of liver disease, alcohol and tobacco consumption, dietary preferences, and history of blood transfusion.

\section{DNA extraction and quantification}

Blood samples were collected on EDTA, stored at $+4{ }^{\circ} \mathrm{C}$ for a maximum of $4 \mathrm{~h}$, and centrifuged $5 \mathrm{~min}$ at $2000 \mathrm{rpm}$. Plasma was then collected and aliquoted. Samples were stored at $-80{ }^{\circ} \mathrm{C}$ before shipment in dry ice.

CfDNA was extracted from a mean volume of $800 \mu \mathrm{L}$ of plasma. Briefly, plasma was digested overnight at $37^{\circ} \mathrm{C}$ under mild agitation in a buffer corresponding to a final concentration of $10 \mathrm{mM}$ EDTA, $2 \%$ SDS, and $0.2 \mu \mathrm{g} / \mathrm{mL}$ proteinase K (Eurobio, Les Ulis, France). Samples were, then, submitted to a phenol-chloroform extraction and ethanol precipitated overnight at $-80{ }^{\circ} \mathrm{C}$ in the presence of sodium acetate and T40 dextran. DNA was solubilized in TE buffer and quantified by Qubit dsDNA HS assay kit (Life Technologies, Villebon, France).

\section{PCR analyses}

Amplifiability of cfDNA was first checked on a singlecopy nuclear gene (MGEA5, meningioma-expressed antigen 5, sense primer, CCCGTACAAAGGAAGATCCA, reverse primer, ATTTGTACCAGGTGGCAAGG). Droplet digital PCR reactions were performed on the QX100 system (Bio-Rad, Hercules, CA, USA). Reaction mixture consisted in $10 \mu \mathrm{l}$ ddPCR Supermix (Bio-Rad, Marnesla-Coquette, France), 1.6X TaqMan ${ }^{\mathrm{TM}}$ SNP Genotyping Assay (C__60493487_10, Life technologies, Villebon, France), and $3 \mu$ of DNA isolated from plasma samples in a final volume of $20 \mu \mathrm{l}$ was mixed with $70 \mu \mathrm{l}$ of droplet generation oil (Bio-Rad) and partitioned into approximately 20,000 droplets in the QX100 droplet generator (Bio-Rad). The droplets generated from each sample were transferred to a 96-well plate, and PCR amplification was conducted in a iCycler PCR instrument (Bio-Rad) with the following condition: $95{ }^{\circ} \mathrm{C}$ for $10 \mathrm{~min}, 40$ cycles of $94{ }^{\circ} \mathrm{C}$ for $30 \mathrm{~s}$ with a ramping of $2 \%, 59^{\circ} \mathrm{C}$ for 1 min with a ramping of $2 \%$, followed by $98{ }^{\circ} \mathrm{C}$ for $5 \mathrm{~min}$ and a hold at $4{ }^{\circ} \mathrm{C}$. After amplification, the 96-well plate was loaded onto the QX100 droplet reader (Bio-Rad) that measures automatically the fluorescence intensity in individual droplets. Generated data were subsequently analyzed with QuantaSoft ${ }^{\mathrm{TM}}$ software (Bio-Rad) based on positive and negative droplet populations. Genomic DNA from the PLC/PRF5 HCC cell line that harbors the R249S mutation of TP53 was included as a positive control in each experiment. In parallel, the genomic DNA extracted from peripheral blood mononuclear cells of a European subject without the R249S mutant was used as negative control in all experiments.

From the initial series, a total of 149 and 164 samples collected from patients with HCC and CLD were suitable for analyses by droplet digital PCR (ddPCR). Samples positive for R249S mutants were tested at least twice in two independent replicates included in independent runs. An identical procedure was used for negative samples yielding less than $1.0 \mathrm{E}+03$ positive droplets for the control locus MGEA5 (cytoband: 10q24.32). Those samples negative for TP53 R249S but yielding more than 1000 compartments positive for $M G E A 5$ were considered as providing sufficient information and tested once. Samples discarded did not generate any fluorescent droplet ( $n=104$ samples) or a sufficient merged number of droplets positive for the reference gene MGEA5 (41 cases, droplet count $=233 \pm 30$ vs. $4000 \pm 249$, $P<0.0001)$. Overall, poorly performing samples were characterized by low cfDNA concentrations (median: 67 vs. $636 \mathrm{ng} / \mathrm{mL}, P<0.0001)$. All cfDNA samples of the 49 subjects with healthy liver have been also submitted to ddPCR. Samples were considered positive for TP53 R249S when at least two droplets were positive for the mutant allele.

\section{Statistical analyses}

Statistical analyses were performed using a Prism 6.0 statistical package. Numerical variables were summarized by their median, mean, and range according to their types of distribution (normal or not). They were compared either by a Student's $T$ test or by a Mann-Whitney test as appropriate. 
Categorical variables were summarized as frequencies that were compared by Fisher's exact test. All tests were twosided, and the level of significance was set at $P<0.05$. Receiver operating curves were produced with the Johns Hopkins University School of Medicine, Baltimore online software application (www.jrocfit.org) [17].

\section{Results}

The clinico-biological features of cases and controls analyzed are provided in Table 1. Briefly, the mean age of subjects included was for all groups in the fifth decade of life span with HCC cases somewhat older than CLD or HL (47 vs. 41-42 years). Males were overrepresented in HCC group when compared with CLD. AFP was measured above the diagnostic level for HCC (>350 ng/mL) in more than half of cases while another $17 \%$ of HCC cases presented a value in a "suspect" range of $10-350 \mathrm{ng} / \mathrm{mL}$. In the current series of patients, AFP levels above $10 \mathrm{ng} / \mathrm{mL}$ were thus strongly associated with liver malignancy $(P=2.2 \mathrm{E}-40$, OR 78.8, 95\% CI 27.9-305.5).

Viral risk factors as assessed through serological markers or molecular techniques were closely similar between HCC and CLD with the notable exception of HBV genotypes as mentioned in our previous works [18].

Among non-infectious risk factors (RFs) of HCC, alcohol abuse and obesity were significantly more frequent in tumors group than in controls. Indeed, the cumulative number of HCC RFs (HBV, HCV, HDV, alcohol, tobacco, obesity, or Type 2 diabetes) was significantly higher in patients with HCC $(1.93 \pm 0.08)$ than in other subgroups $(1.35 \pm 0.05$ in CLD and $0.3 \pm 0.07$ in HL, $P<0.0001$, see Table 1$)$. Regarding nutritional preferences, patients with HCC were more often consumers of millet (genus Pennisetum) flour than subjects included in CLD and HL groups, whereas patients with any liver diseases (HCC, CLD) were more often plantain flour (Musa paradisiaca) consumers than HL. We assessed noninvasively the presence of liver cirrhosis by using cutoffs recommended in three different scoring systems (API, APRI, FIB4). The prevalence of cirrhosis was according to these scores ranging between 29 and 50\%. Symmetrically, the absence of significant fibrosis as suggested by the three indices was estimated around 17-20\%. The survival was known for 80 patients from Yaoundé only. With 17.5 days (interquartile range 8-31), the median survival time was dramatically short indicating that Cameroonian patients are waiting for the very last extremity before seeking for modern medical care.

Both mean and median cfDNA concentrations were significantly decreasing from $\mathrm{HCC}$ to $\mathrm{HL}$ subgroups with CLD occupying an intermediate position (see Fig. 1a). Clinicobiological features associated with high concentrations of cfDNA in the 195 patients with HCC were scarce. An apparently solid determinant of cfDNA concentration was the absolute number of leukocytes per $\mathrm{mL}$ of blood (Fig. 1b). Among the different subclasses of leukocytes, neutrophils count was most tightly linked to cfDNA concentrations. It was not the case, however, for other leukocyte types and liver enzymes (AST is provided as an example), suggesting that cfDNA resulting from hepatocytes destruction usually represents a minor subset of cfDNA molecules even in the context of chronic liver diseases. Interestingly, we observed that samples performing poorly in ddPCR and therefore discarded from subsequent analysis displayed significantly lower neutrophils count than those providing a sufficient ddPCR signal $(3512 \pm 317$ vs. $5356 \pm 389 / \mathrm{mL}, P=0.0128$, not shown). Regarding infectious or nutritional risk factors, anti-HCV carriers displayed a trend for lower cfDNA content than other HCC patients, whereas the reverse was true for maize consumers (not shown).

We next explored cfDNA for the presence of the common g747t mutation resulting in the $\mathrm{R} 249 \mathrm{~S}$ change affecting $\mathrm{p} 53$ DNA-binding domain. To this aim, we used a droplet digital PCR method (see "Methods" section) and retained finally 149 HCC, 164 CLD, and $49 \mathrm{HL}$ ( $n=362$ individuals in total) that yielded enough droplets positive for wild-type allele and consistent fluorescent signal (median droplet number: 2288, interquartile range 1438-4521, range 553-33307). Almost one-fourth of patients with HCC ( $n=37 / 149,24.8 \%)$ were positive for TP53 R249S mutation in cfDNA. This proportion was significantly higher than in patients with CLD $(n=9 / 164,5.5 \%, P=5.5 \mathrm{E}-07$, OR $6.4,95 \%$ CI 2.7-6.5) or healthy controls $(n=3 / 49,6.6 \%, P=0.0037$, OR $5.0,95 \% \mathrm{CI}$ 1.4-26.5, see Fig. 2). No significant difference was observed between any group of subjects from Cameroon or Central African Republic (CAR).

Patients with HCC positive for R249S exhibited distinctive clinico-biological features such as higher AFP values or higher HBV loads and increased cumulative number of hepato-carcinogenic risk factors (HBV, HCV, HDV, alcohol intake, tobacco consumption, obesity, Type 2 diabetes, see Fig. $3 \mathrm{a}-\mathrm{c}$ ). In addition, mutation carriers were characterized by increased total bilirubin values and a skewed blood count with a decreased percentage of circulating lymphocytes (not shown). Likewise, all prognostic scores involving lymphocytes count (neutrophils-to-lymphocytes ratio, platelet-tolymphocytes ratio, lymphocytes-to-monocytes ratio) were worsened in R249S carriers compared with WT (Fig. 3d, e). In addition, neutrophils count and $\gamma$-glutamyltranspeptidaseto-platelet ratio (GPRI) were mildly increased in R249S carriers (Fig. 3f). We did not observe any association of R249S mutation presence with age of patients, region of residence, season of inclusion nor with cfDNA or aminotransferases concentrations. Overall, we observed that the presence of R249S mutant was the hallmark of patients presenting a 
Table 1 Clinical and biological features of the three groups of subjects analysed

\begin{tabular}{|c|c|c|c|c|c|c|}
\hline Clinical features & $\begin{array}{l}\text { HCC } \\
n=195\end{array}$ & $\begin{array}{l}\text { CLD } \\
n=263\end{array}$ & $\begin{array}{l}\mathrm{HL} \\
n=49\end{array}$ & $\begin{array}{l}P \text { value } \\
\text { HCC vs CLD }\end{array}$ & $\begin{array}{l}P \text { value } \\
\text { HCC vs HL }\end{array}$ & $\begin{array}{l}P \text { value } \\
\text { CLD vs HL }\end{array}$ \\
\hline \multicolumn{7}{|l|}{ Age (years) } \\
\hline Mean \pm SD & $47.1 \pm 16.6$ & $41.2 \pm 15.5$ & $42.7 \pm 16.3$ & 0.0013 & ns & ns \\
\hline Range & $14-93$ & $13-77$ & $17-82$ & & & \\
\hline Sex ratio M:F & $2.6(144 / 54)$ & $1.7(168 / 95)$ & $5.1(41 / 8)$ & 0.0451 & $\mathrm{~ns}$ & 0.0076 \\
\hline \multicolumn{7}{|l|}{$\operatorname{AFP}(\mathrm{ng} / \mathrm{mL})$} \\
\hline$>350 \mathrm{ng} / \mathrm{mL}$ & 51.6 & 0.0 & - & & & \\
\hline $10<c<350$ & 17.0 & 1.3 & - & $<0.0001$ & - & - \\
\hline$<10$ & 31.4 & 98.6 & - & & & \\
\hline \multicolumn{7}{|l|}{ Viral markers $(\%)$} \\
\hline $\mathrm{HBsAg}(+)$ & 58.8 & 62.5 & 10.2 & ns & $<0.0001$ & $<0.0001$ \\
\hline Anti-HBc(+) & 89.4 & 96.9 & 34.6 & ns & $<0.0001$ & $<0.0001$ \\
\hline Anti-HBs(+) & 22.3 & 28.9 & 34.6 & ns & ns & ns \\
\hline $\operatorname{HBeAg}(+)$ & 15.9 & 14.9 & - & ns & - & - \\
\hline Anti-delta $(+)$ & 23.4 & 24.8 & 0.0 & ns & $<0.0001$ & $<0.0001$ \\
\hline Anti-HCV(+) & 24.3 & 22.8 & 2.0 & ns & $<0.0001$ & $<0.0001$ \\
\hline Non-BDC & 23.9 & 17.9 & 87.8 & $0.128(\mathrm{~ns})$ & $<0.0001$ & $<0.0001$ \\
\hline HBV loads (Log IU) & $3.2 \pm 0.2$ & $3.4 \pm 0.2$ & - & ns & - & - \\
\hline HBV genotypes A/E & $83 / 17$ & $14 / 86$ & - & $<0.0001$ & - & - \\
\hline HCV loads (Log IU) & $5.1 \pm 0.3$ & $5.2 \pm 0.3$ & - & ns & - & - \\
\hline Anti-HIV & 10.2 & 15.3 & 9.0 & ns & ns & ns \\
\hline Anti-HEV (IgG and/or IgM) & 39.2 & 23.6 & 0.0 & 0.0051 & - & - \\
\hline \multicolumn{7}{|l|}{ Other risk factors of liver tumors (\%) } \\
\hline Alcohol abuse & 51.5 & 24.4 & 10.8 & $<0.0001$ & $<0.0001$ & $0.087(\mathrm{~ns})$ \\
\hline Tobacco use & 9.4 & 6.7 & 6.1 & $\mathrm{~ns}$ & ns & ns \\
\hline Type 2 diabetes & 10.0 & 5.9 & 4.2 & ns & ns & ns \\
\hline Obesity & 10.0 & 2.7 & 0.0 & 0.0033 & 0.0263 & 0.0033 \\
\hline Cumulative number HCC RF & $1.93 \pm 0.08$ & $1.35 \pm 0.05$ & $0.3 \pm 0.07$ & $<0.0001$ & $<0.0001$ & $<0.0001$ \\
\hline \multicolumn{7}{|l|}{ Staple food $(\%)$} \\
\hline Cassava & 55.5 & 58.4 & 42.8 & ns & ns & $0.093(\mathrm{~ns})$ \\
\hline Groundnut & 0.6 & 20.0 & 0.0 & $<0.0001$ & ns & 0.0002 \\
\hline Maize & 29.4 & 27.7 & 18.3 & ns & ns & ns \\
\hline Millet & 52.2 & 26.1 & 20.4 & $<0.0001$ & 0.0001 & ns \\
\hline Plantain & 56.8 & 46.1 & 28.5 & $0.075(\mathrm{~ns})$ & 0.0009 & 0.041 \\
\hline Rice & 5.9 & 9.2 & 6.1 & ns & ns & ns \\
\hline Sorghum & 20.2 & 19.2 & 16.3 & ns & ns & ns \\
\hline \multicolumn{7}{|l|}{ Hematology } \\
\hline Prothrombin time (\%) & $63 \pm 19$ & - & - & - & - & - \\
\hline White blood cells count $(103 / \mathrm{mL})$ & $7.6 \pm 4.9$ & - & - & - & - & - \\
\hline Neutrophils (\%) & $60 \pm 16$ & - & - & - & - & - \\
\hline Monocytes (\%) & $8.8 \pm 4.1$ & - & - & - & - & - \\
\hline Lymphocytes (\%) & $26.7 \pm 13.8$ & - & - & - & - & - \\
\hline Platelets (Giga/mL) & $224 \pm 138$ & - & - & - & - & - \\
\hline Hemoglobin (g/dL) & $10.3 \pm 2.4$ & - & - & - & - & - \\
\hline Blood groups $\mathrm{A} / \mathrm{B} / \mathrm{O} / \mathrm{AB}(\%, n=137)$ & $34 / 23 / 38 / 2$ & - & - & - & - & - \\
\hline $\mathrm{Rh}+(\%)$ & 97.8 & - & - & - & - & - \\
\hline \multicolumn{7}{|l|}{ Biochemistry (mean \pm SEM) } \\
\hline Total bilirubin (mg/L) & $33 \pm 53$ & - & - & - & - & - \\
\hline Conjugated bilirubin (mg/L) & $17 \pm 39$ & - & - & - & - & - \\
\hline Alkaline phosphatase (IU/mL) & $350 \pm 1103$ & - & - & - & - & - \\
\hline
\end{tabular}


Table 1 (continued)

\begin{tabular}{|c|c|c|c|c|c|c|}
\hline Clinical features & $\begin{array}{l}\mathrm{HCC} \\
n=195\end{array}$ & $\begin{array}{l}\text { CLD } \\
n=263\end{array}$ & $\begin{array}{l}\mathrm{HL} \\
n=49\end{array}$ & $\begin{array}{l}P \text { value } \\
\text { HCC vs CLD }\end{array}$ & $\begin{array}{l}P \text { value } \\
\text { HCC vs HL }\end{array}$ & $\begin{array}{l}P \text { value } \\
\text { CLD vs HL }\end{array}$ \\
\hline Aspartate aminotransferase (IU/mL) & $164 \pm 15$ & $186 \pm 57$ & - & 0.105 (ns) & - & - \\
\hline Alanine aminotransferase (IU/mL) & $70 \pm 4$ & $122 \pm 36$ & - & $0.226(\mathrm{~ns})$ & - & - \\
\hline Gamma Glutamyltranspeptidase (IU/mL) & $319 \pm 26$ & - & - & - & - & - \\
\hline ASAT/ALAT ratio & $2.65 \pm 0.17$ & $1.87 \pm 0.12$ & & 0.0002 & - & - \\
\hline \multicolumn{7}{|l|}{ Fibrosis scores } \\
\hline $\mathrm{API}>6$ & 29.3 & - & - & - & - & - \\
\hline APRI $>2.0$ & 38.2 & - & - & - & - & - \\
\hline FIB $4>3.2$ & 50.0 & - & - & - & - & - \\
\hline
\end{tabular}

API age-to-platelet index, APRI AST-to-platelet ratio index, FIB4 fibrosis index based on four factors (ALT, ALT, age, platelets)

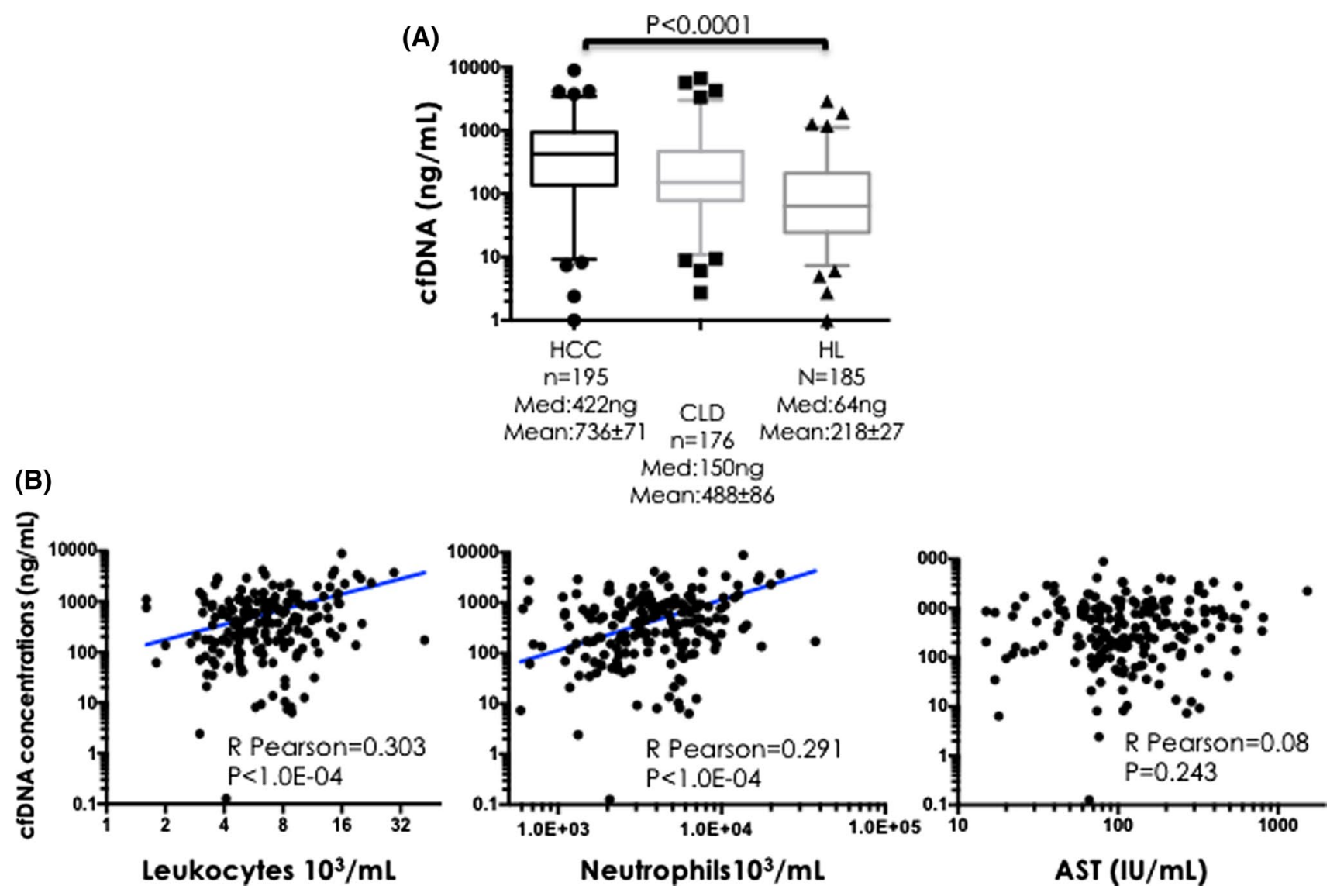

Fig. 1 a Cell-free DNA (cfDNA) concentrations in patients with hepatocellular carcinoma (HCC), chronic liver disease (CLD), and healthy controls (HL). b correlations of cfDNA concentrations with

set of severely disrupted biochemical and hematological features.

The number of R249S carriers in CLD was too small to generate significant differences with wild-type subjects.

We next wonder whether the proportion of droplets positive for R249S out of the total of droplets positive for wild-type allele (R249R) was different in patients with a HCC and in those without any tumor. The leukocytes or neutrophils counts. Aspartate aminotransferase concentrations are not correlated with those of cfDNA

difference observed between these groups was highly significant (mean count of positive droplets $=15.3 \pm 3.7$ vs. $0.5 \pm 0.3 \%, P=7.0 \mathrm{E}-04)$, suggesting that initial R249S concentration or its subsequent evolution might be used as diagnostic biomarker complementary to AFP in Central Africa (Fig. 4a). Indeed, the receiver operating characteristic (ROC) curves drawn with R249S droplet percentage and AFP concentration were rather similar and performing 


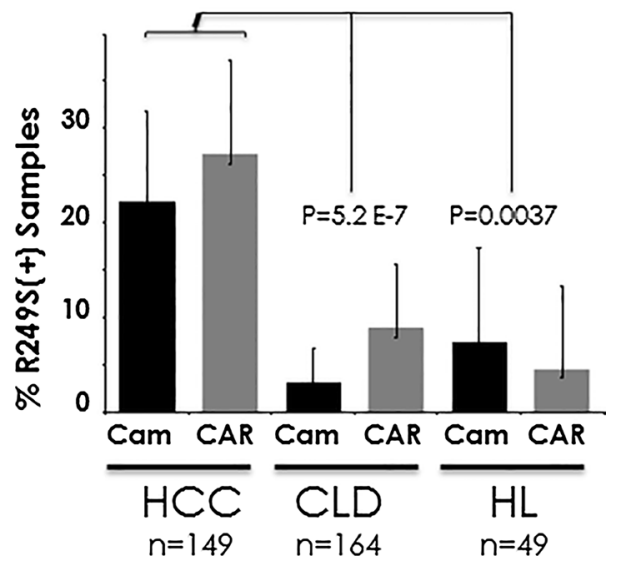

Fig. 2 Prevalence of R249S mutants in patients with HCC, with chronic liver disease (CLD) and in subjects with a healthy liver (HL)

much better than cfDNA concentrations alone (Fig. 4b). Regarding survival of Cameroonian patients, cfDNA concentrations and TP53 R249S were not associated with variation of survival at variance with bilirubin concentrations that were the only clinico-biological parameter significantly associated with overall survival $(P=0.0252$, not shown).

\section{Discussion}

Hepatocellular carcinoma is still a major curse in SSA where it claims the life of more than 37,000 individuals each year. It is, indeed, ranking at the first place among tumors affecting both sexes in SSA [1]. The burdens represented both by persistent hepatitis, and aflatoxin-contaminated staple food consumption is primarily responsible for this worrying situation [19]. Unfortunately, economic and development contexts of national health systems in SSA countries make notoriously difficult to foresee any large-scale improvement of survival for the patients afflicted with HCC. Regardless of such adverse environment, it is more than ever necessary to develop efficient biomarkers (1) to timely diagnose HCC in patients who might benefit from any type of appropriate care and (2) to landscape in a public health approach those subsets of the local populations eventually more exposed to hepatitis viruses or carcinogenic mycotoxins [20,21].

In this context, the recent development of ddPCR technique represents a significant contribution to improve the panel of standardized techniques enabling the detection of rare molecules in human body fluids [22]. Genomic DNA is a robust compound that can be retrieved from altered tissues or ancient remains; it represents, therefore, a good candidate to explore cancer biomarkers in the tropical conditions
(A)

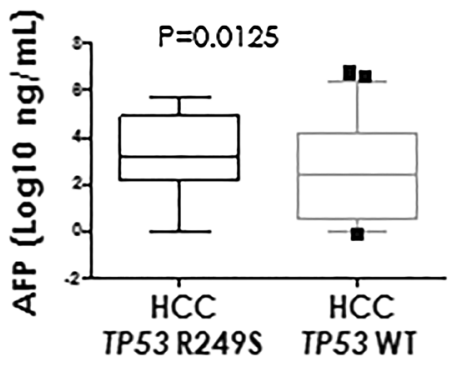

(D)

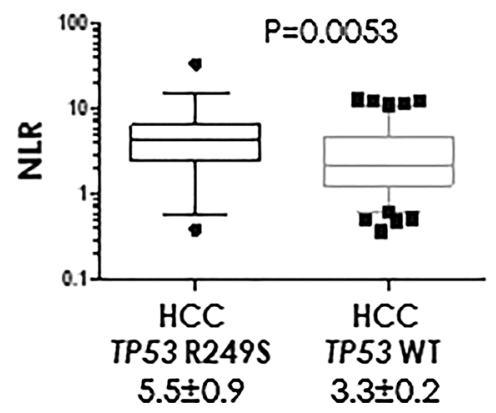

(B)

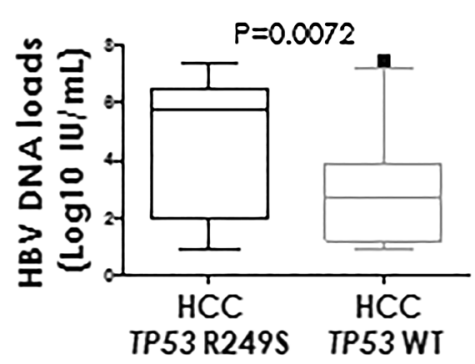

(E)

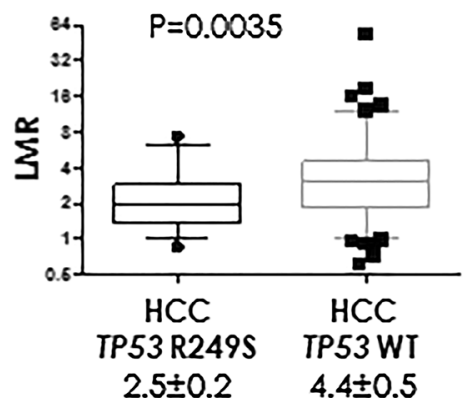

(C)

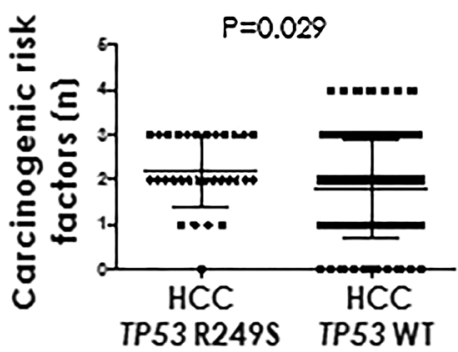

(F)

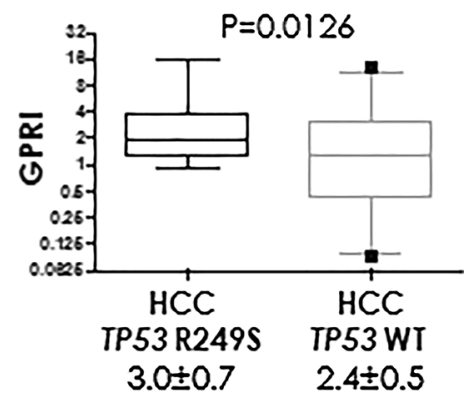

Fig. 3 Clinico-pathological correlations in the presence of R249S. a R249S correlates positively with AFP concentration. b R249S correlates positively with HBV DNA loads. c R249S correlates positively with cumulative number of tumor risk factors in patients. d R249S correlates positively with neutrophils-to-lymphocytes ratio (NLR). e R249S correlates negatively with lymphocytes-to-monocytes ratio (LMR). f R249S correlates positively with $\gamma$-glutamyltranspeptidaseto-platelet ratio (GPRI) 


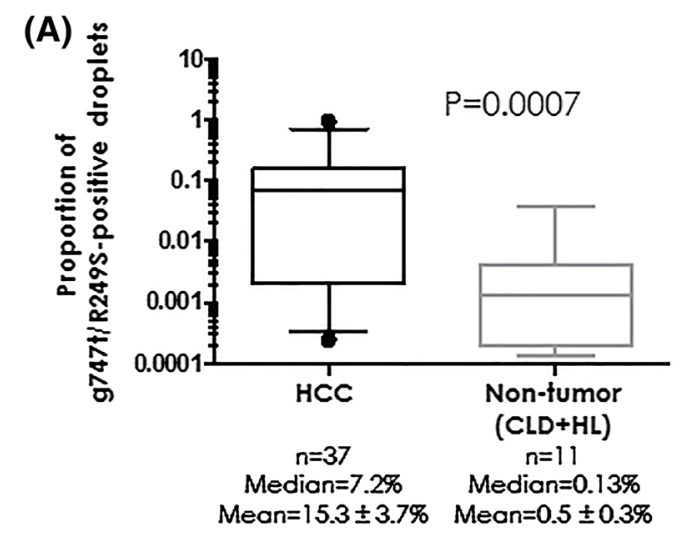

(B)

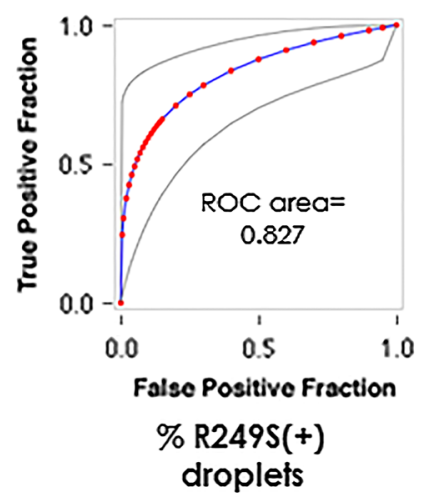

(C)

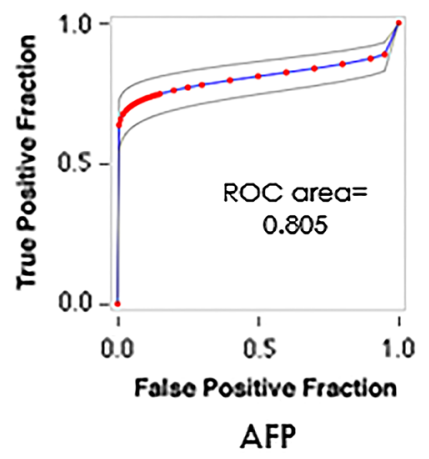

(D)

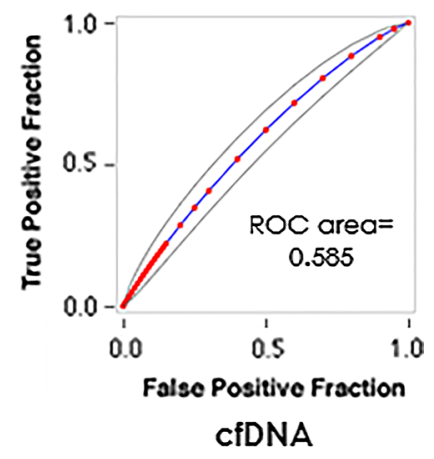

Fig. 4 a Fraction of droplets with R249S in HCC and non-HCC fcDNA samples. b-d Receiver operating curves (ROC) for R249S-poitive fraction, AFP concentration, and cfDNA concentration according to values measured in HCC and CLD

prevailing in SSA [23, 24]. Recent research has shown that the most abundant mutation in human $\mathrm{HCC}(>50 \%$ of cases) affects the TERT gene promoter $(-124 \mathrm{c}>\mathrm{t})$ [25]. Unfortunately located in a GC-rich and highly repetitive region, this frequent alteration is almost impossible to detect with reasonable confidence from small-sized DNA fragments that compose cfDNA (A. Marchio, unpublished results). We, thus, focused on the presence in cfDNA of the well-known g747t/R249S mutations that affect TP53 [10, 20]. This alteration attributed to the mutagenic activity of AFB 1 is known to be prevalent in HCC from patients living in the inter/subtropical areas albeit with a remarkably extensive variability [26-28]. R249S is frequently detected in tumors from Eastern Asia and SSA but curiously more rarely found in HCC from patients coming from Southern Asia or South America where the presence of AFB1 in staple food is not unusual though [29]. This intriguing inconsistency is attributed both to variations in real AFB1 exposure due to nutritional or cooking practices and to genetic polymorphisms in enzymes controlling AFB1 detoxification [30]. R249S was already searched using various techniques from cfDNA of patients with HCC living in North Africa (Egypt), West Africa (Gambia, Nigeria, Mali), and in South Africa [31-33]. Its presence was to our knowledge never investigated from patients living in the vast region designated as Middle Africa by the World Health Organization. The presence of almost $25 \%$ of R249S carriers in patients with HCC and 3-9\% of carriers among non-HCC subjects with or without liver diseases substantiates the recently published surveys reporting the presence of aflatoxins in Cameroon where it has been found in various body fluids including those of patients with HCC [34-36]. There were no data available about AFB1 in Central African Republic. The prevalence of R249S mutants in patients from Middle Africa with HCC is apparently in the range of previous observations made in South Africa or Egypt but significantly lower than the figures recurrently published from the Gambia or the Mali although the two latter values result from the use of a different technological approach (Fig. 5) [31, 32, 37-39]. It seems, thus, plausible that the mutagenic pressure applied by AFB1 on populations is higher in West Africa than anywhere else on the continent explaining partly the local high HCC incidence.

Interestingly, the median fractions of mutated alleles in patients with HCC (7\%) and in controls $(0.3 \%)$ are significantly different in accordance with results previously published in the Gambia (11 and $<3 \%$, respectively) [38]. 
Fig. 5 R249S mutant prevalence in bloodstream of patients with HCC in Africa according to the current literature (see "Discussion" section, percentages represent proportions of positive samples)

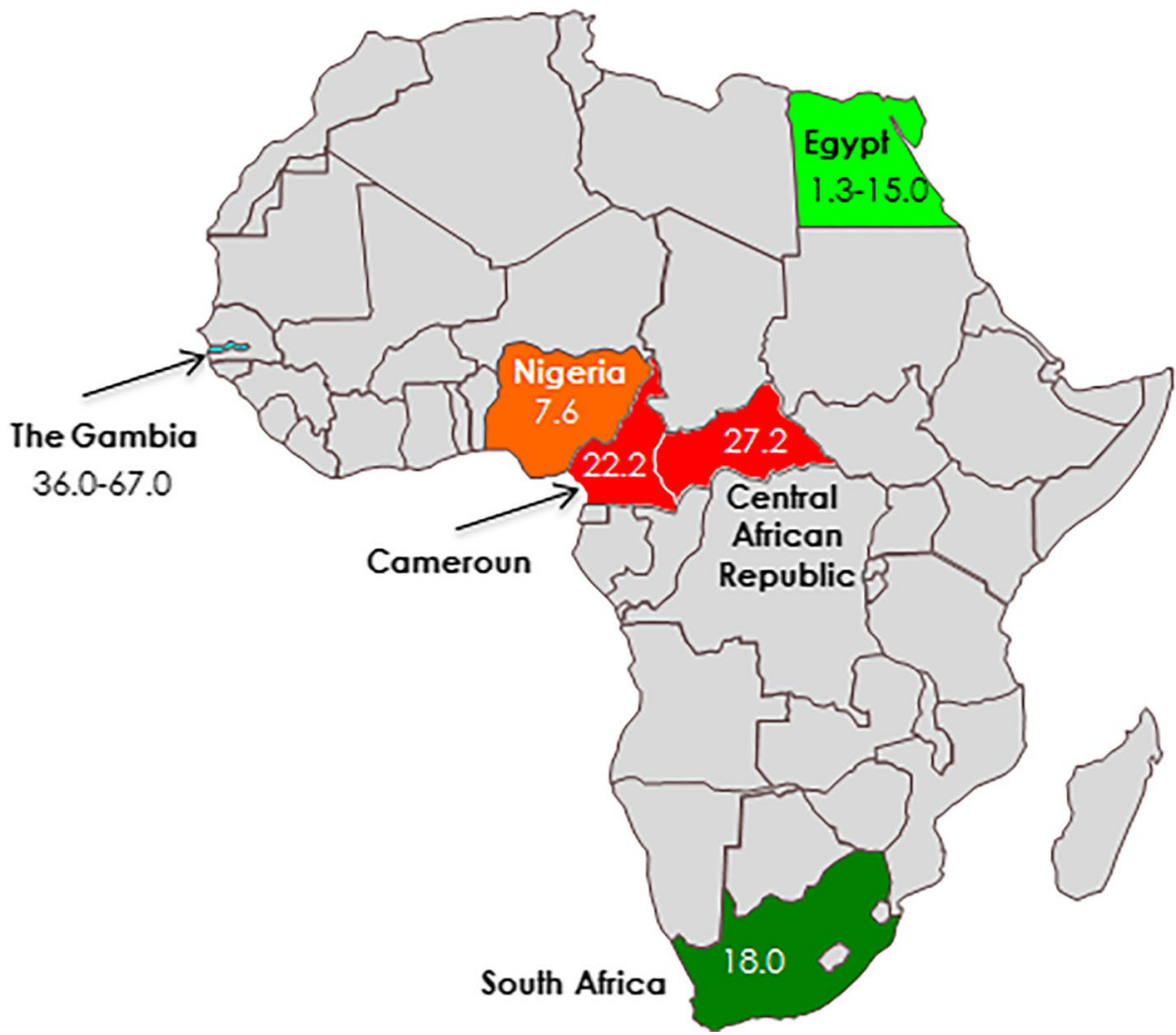

It appears, thus, that the ddPCR technique might bring substantial progress for the field of HCC in SSA. However, the proportion of samples $(n=145 / 458,31.5 \%)$ that did not yield any droplets or generated too few of them represents an issue of the current work. Several solutions to remediate this situation should be considered in the future. Of course, increasing the volume of plasma used for extraction to $2-5 \mathrm{~mL}$ appears as a logical move since samples with low cfDNA concentrations were those that underperformed in ddPCR. A second improvement could be the use of a specific preservation solution for blood collection [40]. Such optimization may increase cfDNA stability and subsequent results especially in tropical conditions. The third variable to increase cfDNA yield and quality is the extraction method. The comparison of precipitation method (used in the current work) with commercial spin columns was, however, not detrimental to precipitation [41, 42]. Notably, considerable variations of cfDNA yields have been observed with spin columns [43]. In the absence of consensus, it is therefore difficult to recommend an extraction method rather than another.

\section{Conclusions}

These observations suggest that the follow-up of R249S fraction in patients with CLD might represent a useful biomarker to diagnose early steps of tumor development. An appropriate use of ddPCR might, therefore, provide a useful time window for subsequent therapeutic intervention. In this regard, recently developed percutaneous approaches that have been shown to be efficient to extend patients life span and applicable in the settings of SSA health systems are promising [44, 45]. Upstream of its clinical use, public health might take advantage of ddPCR development as well. It is indeed conceivable that national health authorities proceed at regular intervals to surveys monitoring the presence of R249S in the bloodstream of populations at risk. Such assessments may result in precise cartography enabling subsequent implementation of measures preventing exposure of vulnerable populations to AFB1.

Acknowledgements We express our gratitude to Flore-Ghislaine Mela in CPC of Yaoundé for her help in the collection of clinico-biological data, to Dominique Poveda (Laboratoires CERBA) for his logistic support, to David Laurent for technical assistance, to Marion Benabou and Thomas Bourgeron (Unité de Génétique humaine et fonctions cognitives), and to Nathalie Jolly for guidance to establish a protocol in conformity with french regulations. We feel indebted to Prof. Anne Dejean for her constant support in the laboratory.

Authors' contribution RN, CB, NPK and AM, and PP designed the study. AM, MAA, CB, and PP performed the experiments. $\mathrm{CB}, \mathrm{AB}$, and MAA collected and organized the clinical data. PP performed statistical analysis. PAP, DNN, SMCP provided the clinical data. PP drafted the manuscript. AM, RN, CB, MAA contributed in editing the manuscript. All the authors have read and approved the final version. 
Funding This work was supported by Institut Pasteur International Network (Action Concertée Inter-Pasteurienne No17-2010, and Grant Dedonder), by the direction of Research Applications and Relations with the Industry (VALO Express), and by the French Ligue Nationale contre le Cancer (Equipe labélisée d'Anne Dejean).

\section{Compliance with ethical standards}

Conflict of interest The authors declare that they have no conflict of interests

Ethics approval All procedures performed in studies involving human participants were in accordance with the ethical standards of the institutional and/or national research committee and with the 1964 Helsinki declaration and its later amendments or comparable ethical standards. It was approved by the National Ethics Committee and the Ministry of Health of Cameroon (AAR\#631-00.12) as well as by the Ethics Committee of the Bangui Medical School (No. 10/UB/FACSS/CSVPR/11).

Informed consent Written informed consent was obtained from all the patients (or their parents, in the case of the nine minors between 13 and 17 years old).

Availability of data and materials Data could be available on reasonable request. The minute amount of cell-free DNA present in the plasma of human beings makes impossible the availability of material. Furthermore, according to the French regulations, the consent to exploit the samples was given by the participants to the initial members of the consortium and was not intended to be spread anywhere else.

Open Access This article is distributed under the terms of the Creative Commons Attribution 4.0 International License (http://creativeco mmons.org/licenses/by/4.0/), which permits unrestricted use, distribution, and reproduction in any medium, provided you give appropriate credit to the original author(s) and the source, provide a link to the Creative Commons license, and indicate if changes were made.

\section{References}

1. GLOBOCAN 2012 v1.0, Cancer incidence and mortality worldwide: IARC CancerBase No. 11 [Internet]. [database on the Internet]. International Agency for Research on Cancer. 2013.

2. Higginson J. The geographical pathology of primary liver cancer. Cancer Res. 1963;23:1624-33.

3. Wu F, Khlangwiset P. Health economic impacts and cost-effectiveness of aflatoxin reduction strategies in Africa: case studies in biocontrol and postharvest interventions. Food Addit Contam. 2010;27:496-509.

4. Ott J, Stevens G, Wiersma S. The risk of perinatal hepatitis B virus transmission: hepatitis B e antigen ( $\mathrm{HBeAg}$ ) prevalence estimates for all world regions. BMC Infect Dis. 2012;12:131.

5. Yang J, Mohamed EA, AbdelAziz A, Shousha H, Hashem M, Nabeel M, et al. Characteristics, management, and outcomes of patients with hepatocellular carcinoma in Africa: a multicountry observational study from the Africa Liver Cancer Consortium. Lancet Gastroenterol Hepatol. 2017;2:103-11.

6. Bressac B, Kew M, Wands J, Ozturk M. Selective G to T mutations of p53 gene in hepatocellular carcinoma from southern Africa. Nature. 1991;350:429-31.

7. Pineau P, Marchio A, Battiston C, Cordina E, Russo A, Terris $\mathrm{B}$, et al. In human hepatocellular carcinoma, chromosome instability depends on p53 status and aflatoxin exposure. Mutat Res. 2008;653:6-13.

8. Tanase A, Marchio A, Dumitrascu T, Dima S, Herlea V, Oprisan $\mathrm{G}$, et al. Mutation spectrum of hepatocellular carcinoma from Eastern-European patients betrays the impact of a complex exposome. J Exp Sci Environ Epidemiol. 2014;25:256-63.

9. Marchio A, Cerapio JP, Ruiz E, Cano L, Casavilca S, Terris B, et al. Early forms of liver cancer in South America associate with low hepatitis B virus DNA burden (in revision).

10. Gouas D, Shi H, Hainaut P. The aflatoxin-induced TP53 mutation at codon 249 (R249S): Biomarker of exposure, early detection and target for therapy. Cancer Lett. 2009;286:29-37.

11. Mandel P, Métais P. Les acides nucléiques du plasma sanguin chez l'Homme. CR Soc Biol. 1948;142:241-3.

12. Chan K, Lo Y. Circulating tumour-derived nucleic acids in cancer patients: potential applications as tumour markers. Br J Cancer. 2007;96:681-5.

13. Pathak A, Bhutani M, Kumar S, Mohan A, Guleria R. Circulating cell-free DNA in plasma/serum of lung cancer patients as a potential screening and prognostic tool. Clin Chem. 2006;52:1833-42.

14. Bettegowda C, Sausen M, Leary R, Kinde I, Wang Y, Agrawa N, et al. Detection of circulating tumor DNA in early- and late-stage human malignancies. Sci Transl Med. 2014;6:224ra24.

15. Thierry A, Mouliere F, El Messaoudi S, Mollevi C, Lopez-Crapez $\mathrm{E}$, Rolet $\mathrm{F}$, et al. Clinical validation of the detection of KRAS and BRAF mutations from circulating tumor DNA. Nat Med. 2014;20:430-5.

16. Ladep N, Lesi O, Mark P, Lemoine M, Onyekwere C, Afihene M, et al. Problem of hepatocellular carcinoma in West Africa. World J Hepatol. 2014;6:783-92.

17. Eng J. ROC analysis: web-based calculator for ROC curves. Baltimore: Johns Hopkins University; 2014.

18. Amougou Atsama M, Noah Noah D, Fewou Moundipa P, Pineau $\mathrm{P}$, Njouom R. A prominent role of Hepatitis D Virus in liver cancers documented in Central Africa. BMC Infect Dis. 2016;16:647.

19. Wild C, Montesano R. A model of interaction: aflatoxins and hepatitis viruses in liver cancer aetiology and prevention. Cancer Lett. 2009;286:22-8.

20. Montesano R, Hainaut P, Wild C. Hepatocellular carcinoma: from gene to public health. J Natl Cancer Inst. 1997;89:1844-51.

21. Hussain S, Harris C. p53 mutation spectrum and load: the generation of hypotheses linking the exposure of endogenous or exogenous carcinogens to human cancer. Mutat Res. 1999;428:23-32.

22. Olmedillas-López S, García-Arranz M, García-Olmo D. Current and emerging applications of droplet digital PCR in oncology. Mol Diagn Ther. 2017;21:493-510.

23. Kuś M, Ossowski A, Zielińska G. Comparison of three different DNA extraction methods from a highly degraded biological material. J Forens Leg Med. 2016;40:47-53.

24. Feldman M, Hershkovitz I, Sklan E, Kahila Bar-Gal G, Pap I, Szikossy I, et al. Detection of a tumor suppressor gene variant predisposing to colorectal cancer in an 18th century Hungarian mummy. PLoS ONE. 2016;11:e0147217.

25. Schulze K, Imbeaud S, Letouzé E, Alexandrov L, Calderaro J, Rebouissou S, et al. Exome sequencing of hepatocellular carcinomas identifies new mutational signatures and potential therapeutic targets. Nat Genet. 2015;47:505-11.

26. Kirk G, Camus-Randon A, Mendy M, Goedert J, Merle P, Trepo C, et al. Ser-249 p53 mutations in plasma DNA of patients with hepatocellular carcinoma from The Gambia. J Natl Cancer Inst. 2000;92:148-53.

27. Jackson P, Qian G, Friesen M, Zhu Y, Lu P, Wang J, et al. Specific p53 mutations detected in plasma and tumors of hepatocellular carcinoma patients by electrospray ionization mass spectrometry. Cancer Res. 2001;61:33-5. 
28. Unsal H, Yakicier C, Marçais C, Kew M, Volkmann M, Zentgraf $\mathrm{H}$, et al. Genetic heterogeneity of hepatocellular carcinoma. Proc Natl Acad Sci USA. 1994;91:822-6.

29. Marchio A, Bertani S, Rojas-Rojas T, Doimi F, Terris B, Deharo E, et al. A peculiar mutation spectrum emerging from young Peruvian patients with hepatocellular carcinomas. PLoS ONE. 2014;9:e114912.

30. Zhang Y. Interactions of chemical carcinogens and genetic variation in hepatocellular carcinoma. World J Hepatol. 2010;2:94-102.

31. Traoré F, Gormally E, Villar S, Friesen M, Groopman J, Vernet $\mathrm{G}$, et al. Molecular characteristics of Hepatitis B and chronic liver disease in a cohort of HB carriers from Bamako, Mali. BMC Infect Dis. 2015;15:180.

32. Kimbi G, Kew M, Yu M, Arakawa K, Hodkinson J. 249ser p53 mutation in the serum of black southern African patients with hepatocellular carcinoma. J Gastroenterol Hepatol. 2005;20:1185-90.

33. Igetei R, Otegbayo J, Ndububa D, Lesi O, Anumudu C, Hainaut $\mathrm{P}$, et al. Detection of p53 codon 249 mutation in Nigerian patients with hepatocellular carcinoma using a novel evaluation of cellfree DNA. Ann Hepatol. 2008;7:339-44.

34. Tchana A, Moundipa P, Tchouanguep F. Aflatoxin contamination in food and body fluids in relation to malnutrition and cancer status in Cameroon. Int J Environ Res Public Health. 2010;7:178-88.

35. Njumbe Ediage E, Diana Di Mavungu J, Song S, Sioen I, De Saeger S. Multimycotoxin analysis in urines to assess infant exposure: a case study in Cameroon. Environ Int. 2013;58:50-9.

36. Abia W, Warth B, Sulyok M, Krska R, Tchana A, Njobeh P, et al. Bio-monitoring of mycotoxin exposure in Cameroon using a urinary multi-biomarker approach. Food Chem Toxicol. 2013;62:927-34.
37. Kirk G, Lesi O, Mendy M, Szymañska K, Whittle H, Goedert J, et al. 249(ser) TP53 mutation in plasma DNA, hepatitis B viral infection, and risk of hepatocellular carcinoma. Oncogene. 2005;24:5858-67.

38. Lleonart M, Kirk G, Villar S, Lesi O, Dasgupta A, Goedert J, et al. Quantitative analysis of plasma TP53 249Ser-mutated DNA by electrospray ionization mass spectrometry. Cancer Epidemiol Biomark Prev. 2005;14:2956-62.

39. El-Din H, Ghafar N, Saad N, Aziz M, Rasheed D, Hassan E. Relationship between codon 249 mutation in exon 7 of p53 gene and diagnosis of hepatocellular carcinoma. Arch Med Sci. 2010;3:348-55.

40. Parpart-Li S, Bartlett B, Popoli M, Adleff V, Tucker L, Steinberg $\mathrm{R}$, et al. The effect of preservative and temperature on the analysis of circulating tumor DNA. Clin Cancer Res. 2017;23:2471-7.

41. Xue X, Teare M, Holen I, Zhu Y, Woll P. Optimizing the yield and utility of circulating cell-free DNA from plasma and serum. Clin Chim Acta. 2009;404:100-4.

42. Lu J, Liang Z. Circulating free DNA in the era of precision oncology: pre- and post-analytical concerns. Chron Dis Transl Med. 2016;2:223-30.

43. Fleischhacker M, Schmidt B, Weickmann S, Fersching D, Leszinski G, Siegele B, et al. Methods for isolation of cellfree plasma DNA strongly affect DNA yield. Clin Chim Acta. 2011;412:2085-8.

44. Poulou L, Botsa E, Thanou I, Ziakas P, Thanos L. Percutaneous microwave ablation vs radiofrequency ablation in the treatment of hepatocellular carcinoma. World J Hepatol. 2015;7:1054-63.

45. McWilliams J, Yamamoto S, Raman S, Loh C, Lee E, Liu D, et al. Percutaneous ablation of hepatocellular carcinoma: current status. J Vasc Interv Radiol. 2010;21:S204-13. 